\title{
Repeated surveys and the Kalman filter*
}

\author{
Jo Thori Lind ${ }^{\dagger}$
}

March 23, 2005

\begin{abstract}
The time series nature of repeated surveys is seldom taken into account. The few studies that do so usually smooth the period-wise estimates without using the cross sectional information. This leads to inefficient estimation. We present a statistical model of repeated surveys and construct a computationally simple estimator based on the Kalman filter algorithm. The method efficiently uses the whole underlying data set, but only the first and second moments of the data are required for computational purposes.
\end{abstract}

Keywords: Surveys, Kalman filter, time series

JEL Classification: C22, C53, C81

*I wish to thank Jørgen Aasness, Eivind Bernhardsen, Knut R. Wangen, the editor and an anonymous referee for extremely helpful and valuable comments.

${ }^{\dagger}$ Department of Economics, University of Oslo, PB 1095 Blindern, 0317 Oslo, Norway. Email: j.t.lind@econ.uio.no 


\section{Introduction}

A number of statistical series are estimated on the basis regularly repeated surveys. The most common approach is to publish parameter estimates at regular intervals, say each year, pooling surveys collected throughout the year but ignoring previous years. As it is natural to assume that most parameters of interest evolve slowly and smoothly, this is an inefficient use of the data.

Jessen (1942) was the first to suggest to use times series techniques to improve the results from repeated surveys. It was studied in more detail by Gurney and Daly (1965), and the methodology was further improved by Blight and Scott (1973) and Scott and Smith (1974) who suggest using statistical signal extraction methods to filter the time specific estimates of the parameters of interest. See e.g. the survey by Binder and Hidiroglou (1988) for further details on subsequent developments within this tradition. A more general theory of signal extraction using the Kalman filter was suggested by Tam (1987) and further developed by e.g. Binder and Dick (1989), Harvey and Chung (2000), and Pfeffermann (1991).

Their approach is to estimate a parameter, such as the mean, on each individual survey and then apply the Kalman filter on the estimates. However, there is an important loss of efficiency as a lot of information contained in each cross section may be lost by this two step procedure. A more satisfactory approach is to integrate the time series model and the modelling of the individual observations at each period.

We suggest a model where the parameters of interest evolve smoothly over time, and where each observed data point is a noisy observation of the parameter of interest. If we use the ordinary Kalman filter algorithm on such data, this requires inversion of matrices of dimension proportional to the sample size of each survey. This may be computationally 
costly and lead to numerical instability unless each survey is very small. An important improvement is obtained by sequential processing of each element of the observation vector as first suggested by Anderson and Moore (1979, Section 6.4) and further developed by Durbin and Koopman (Durbin and Koopman 2001, Koopman and Durbin 2000, 2003). However, the computational burden of this technique is also increasing in the sample size of the surveys.

In the present work we first show how repeated surveys may be written conveniently on state-space form. We then show how to extend the Kalman filter to obtain an algorithm whose computational burden is constant in the sample size of each survey. Using the same approach, an expression for the likelihood of the data, which we need to estimate unknown hyper parameters, is derived. It turns out that for computational purposes, we only need the empirical first and second moments in each period, so both the computational burden and the data requirements are small. The estimator is also extended to allow for heterogeneity between groups of individuals.

Comparisons of the computation time required for both filtering and calculation of the likelihood function shows that even for surveys of moderate size (about five), the algorithm presented herein is more efficient than both the traditional Kalman filter technique and the univariate approach.

\section{A simple model}

We first consider a simplified univariate version of the model and show how the Kalman filter may be applied to this model. A more general model will be presented in Section 4 . At a survey date $t \in(1, \ldots, T)$ we observe $N_{t}$ individuals. Let $y_{i t}$ denote the observations 
on individual $i$ at time $t$. At the time being, $y_{t}$ is treated as a scalar. Focus is on estimating averages of the $y_{i t}$ 's. We may write

$$
y_{i t}=\mu_{t}+\varepsilon_{i t}
$$

where the mean $\mu_{t}$ is the variable of interest and $\varepsilon_{i t} \sim N\left(0, \sigma_{t}^{2}\right)$ contain individual unobserved characteristics and possible sampling errors. The $\varepsilon_{i t} \mathrm{~s}$ are independent both within and between surveys.

Assume that there is a $n$-vector $\alpha_{t}$ following a linear Markov process

$$
\alpha_{t}=F \alpha_{t-1}+\xi_{t}
$$

where $\xi_{t} \sim N\left(\mathbf{0}_{n \times 1}, Q\right)$ and $F$ is a $n \times n$ transition matrix, such that $\mu_{t}$ follows the observation equation $\mu_{t}=Z \alpha_{t}$ where $Z$ is $1 \times n$. Here $\mathbf{0}_{n \times 1}$ denotes the $n$-dimensional zero vector. Defining the stacked matrices $\tilde{y}_{t}=\left(y_{1 t}, \ldots, y_{N_{t}}\right)^{\prime}$ and $\tilde{\varepsilon}_{t}=\left(\varepsilon_{1 t}, \ldots, \varepsilon_{N_{t} t}\right)^{\prime}$ we can write

$$
\tilde{y}_{t}=\iota_{N_{t}} Z \alpha_{t}+\tilde{\varepsilon}_{t}, \quad \tilde{\varepsilon}_{t} \sim N\left(\mathbf{0}_{N_{t} \times 1}, \sigma_{t}^{2} I_{N_{t}}\right)
$$

where $\iota_{N_{t}}$ denotes a $N_{t}$-dimensional unit vector. If we treat $\iota_{N_{t}} Z$ as a single matrix transforming the state vector into the expectation of the observed data, we see that this is a model in state space form ${ }^{1}$, and given suitable assumptions on the distribution of $\alpha_{0}$, we can in principle use standard techniques.

\section{The Kalman filter}

If we know the parameters of the model, an optimal estimate of the $\alpha$ 's and the $\mu$ 's may be calculated by means of the Kalman filter (see e.g. Durbin and Koopman (2001), Harvey

\footnotetext{
${ }^{1}$ The dimension of $\tilde{y}_{t}$ usually varies with time, but it is easily shown that this does not cause any difficulties (Durbin and Koopman 2001: Section 4.10)
} 
(1989), or Hamilton (1994: Ch. 13)). At date $t$, the information set is $\mathcal{Y}_{t}=\left(\tilde{y}_{1}, \ldots, \tilde{y}_{t}\right)^{\prime}$. Let us denote the expectation and the covariance matrix of the vector $\alpha_{t_{1}}$ at date $t_{1}$ given the information set at date $t_{2}$ as

$$
\begin{aligned}
a_{t_{1} \mid t_{2}} & \equiv E\left(\alpha_{t_{1}} \mid \mathcal{Y}_{t_{2}}\right) \\
V_{t_{1} \mid t_{2}} & =E\left[\left(\alpha_{t_{1}}-a_{t_{1} \mid t_{2}}\right)\left(\alpha_{t_{1}}-a_{t_{1} \mid t_{2}}\right)^{\prime} \mid \mathcal{Y}_{t_{2}}\right]
\end{aligned}
$$

The Kalman filter is calculated by the following recursion:

$$
\begin{aligned}
a_{t \mid t-1} & =F a_{t-1 \mid t-1} \\
V_{t \mid t-1} & =F V_{t-1 \mid t-1} F^{\prime}+Q \\
a_{t \mid t} & =a_{t \mid t-1}+K_{t}\left(\tilde{y}_{t}-\iota_{N_{t}} Z a_{t \mid t-1}\right) \\
V_{t \mid t} & =V_{t \mid t-1}-K_{t} \iota_{N_{t}} Z V_{t \mid t-1} \\
K_{t} & =V_{t \mid t-1} Z^{\prime} \iota_{N_{t}}^{\prime}\left(\iota_{N_{t}} Z V_{t \mid t-1} Z^{\prime} \iota_{N_{t}}^{\prime}+\sigma_{t}^{2} I_{N_{t}}\right)^{-1} .
\end{aligned}
$$

The two first equations are straightforward to calculate. However, in its current form, (4) includes $\left(\iota_{N_{t}} Z V_{t \mid t-1} Z^{\prime} \iota_{N_{t}}^{\prime}+\sigma_{t}^{2} I_{N_{t}}\right)^{-1}$. This is an inversion of a $N_{t} \times N_{t}$-matrix which requires large amounts of calculation if the sample size $N_{t}$ is large. However, due to the data structure assumed above, (4) may be written as

$$
\begin{aligned}
V_{t \mid t} & =\left(V_{t \mid t-1}^{-1}+\sigma_{t}^{-2} N_{t} Z^{\prime} Z\right)^{-1} \\
a_{t \mid t} & =a_{t \mid t-1}+\sigma_{t}^{-2} N_{t} V_{t \mid t} Z^{\prime}\left(\bar{y}_{t}^{G}-Z a_{t \mid t-1}\right)
\end{aligned}
$$

where $\bar{y}_{t}$ denotes the average of $y_{i t}$. This recursion only requires inverting matrices of dimension $n \times n$, and is hence computationally less demanding than the traditional recursion (4). The proof, which is based on the matrix inversion lemma, is given for the more general structure in Section 4. 
Using the recursion (5), we calculate estimates of $\alpha_{t}$ given the information set $\mathcal{Y}_{t}$. To obtain efficient estimates of the states $\mu_{i t}$ we should employ the full information set $\mathcal{Y}_{T}$. This is most efficiently carried out by the classical smoother, see e.g. Anderson and Moore (1979: Ch. 7), because it only requires the inversion of $V_{t \mid t-1}$ which is of dimensionality $n$, usually a small number. The fixed interval smoother of de Jong (1989) and Kohn and Ansley (1989), which is computationally more efficient in the standard case, requires the inversion of $\left(\iota_{N_{t}} Z V_{t \mid t-1} Z^{\prime} \iota_{N_{t}}^{\prime}+\sigma_{t}^{2} I_{N_{t}}\right)$, which is of dimension $N_{t} \times N_{t}$ in each recursion. As $N_{t}$ is usually a large number, this implies a heavier computational burden, so this approach will usually be inefficient within this class of models.

\section{A general model}

Before discussing estimation of the parameters of the model, we set up a more general model that allows for vectors of observed variables and group-wise heterogeneity between observed individuals.

Now $y_{i t}$ denote the $m$-vector of observations on individual $i$ at time $t$. Again, we can write $y_{i t}=\mu_{i t}+\varepsilon_{i t}$ where $\varepsilon_{i j} \sim N\left(\mathbf{0}_{m \times 1}, \Sigma_{t}\right)$. It is normally not particularly interesting to estimate a separate $\mu$ for every individual. Above the $\mu_{i t}$ 's were assumed to be the same for all individuals at a particular date. But it is often fruitful to group individuals into e.g. geographical regions or household types, and allow the groups to have different $\mu \mathrm{s}$. This is the approach we will pursue now. Assume that there are $G$ such groups, and an associated $\mu_{g t}$ for all $g \in(1, \ldots, G)$ at every date. ${ }^{2}$ It will be useful to consider the stacked vector of

\footnotetext{
${ }^{2}$ The covariance matrix $\Sigma_{t}$ is assumed the be identical for every group, but this assumption is easily relaxed.
} 
group means at date $t$

$$
\mu_{t}=\left(\mu_{1 t}^{\prime}, \ldots, \mu_{G t}^{\prime}\right)^{\prime}
$$

The observation equation may now be written as

$$
y_{i t}=J_{g(i)} \mu_{t}+\varepsilon_{i t}
$$

where $g$ is the function that associates to each individual $i$ the group that it belongs to, and $J_{g t}$ the selection matrix

$$
J_{g}=\left(\begin{array}{lllll}
\mathbf{0}_{(g-1) m \times m} & \vdots & I_{m} & \vdots & \mathbf{0}_{(G-g) m \times m}
\end{array}\right),
$$

which selects the appropriate elements from the vector $\mu_{t}$ for individuals in group $g$.

As above, $\alpha_{t}$ follows a linear Markov process (2) and $\mu_{t}=Z \alpha_{t}$, so $Z$ translates $\alpha_{t}$ into each group's vector of means. This structure allows for some components, e.g. seasonals, to be identical across groups and others to be group specific.

Defining the stacked matrices $\tilde{J}_{t}=\left(J_{g(1)}^{\prime}, \ldots, J_{g\left(N_{t}\right)}^{\prime}\right)^{\prime}, \tilde{\varepsilon}_{t}=\left(\varepsilon_{1 t}^{\prime}, \ldots, \varepsilon_{N_{t} t}^{\prime}\right)^{\prime}$, and $\tilde{y}_{t}=$ $\left(y_{1 t}^{\prime}, \ldots, y_{N_{t} t}^{\prime}\right)^{\prime}$, we can write the observation equation as

$$
\tilde{y}_{t}=\tilde{J}_{t} Z \alpha_{t}+\tilde{\varepsilon}_{t}, \quad \tilde{\varepsilon}_{t} \sim N\left(\mathbf{0}_{N_{t} m \times 1}, I_{N_{t}} \otimes \Sigma_{t}\right)
$$

In this model, the Kalman filter is calculated by the recursion (4) with $\iota_{N_{t}}$ replaced by $\tilde{J}_{t}$ and $\sigma_{t}^{2} I_{N_{t}}$ by $I_{N_{t}} \otimes \Sigma_{t}$. This implies inverting a matrix of dimension $m N_{t} \times m N_{t}$, but the data structure allows us to write it as

$$
\begin{aligned}
& V_{t \mid t}=\left[V_{t \mid t-1}^{-1}+Z^{\prime}\left(\mathcal{N}_{t}^{G} \otimes \Sigma_{t}^{-1}\right) Z\right]^{-1} \\
& a_{t \mid t}=a_{t \mid t-1}+V_{t \mid t} Z^{\prime}\left(\mathcal{N}_{t}^{G} \otimes \Sigma_{t}^{-1}\right)\left(\bar{y}_{t}^{G}-Z a_{t \mid t-1}\right) .
\end{aligned}
$$

In these expressions, $\bar{y}_{t}^{G}$ denotes the within group averages defined as

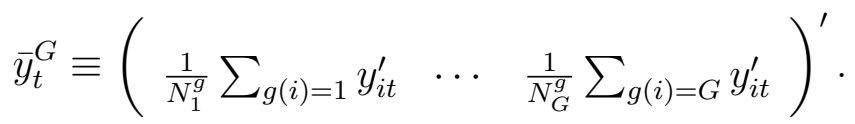


The matrix $\mathcal{N}_{t}^{G}$ is the $G \times G$ matrix with the number of members of each group at date $t$ along the diagonal. The proof is found in the Appendix.

\section{Estimation}

The filtering algorithm described above was based upon the knowledge of the parameters of the model. Since they are normally not known, they will have to be estimated. The usual approach to estimating parameters in Kalman filter models is maximum likelihood. ${ }^{3}$ The unknown parameters are contained in some vector $\Theta$, so the matrices $F$ and $Z$ and the covariance matrices $\Sigma_{t}$ and $Q$ are known functions of $\Theta$. The likelihood of the data is

$$
f\left(\mathcal{Y}_{T} ; \Theta\right)=f\left(\tilde{y}_{1}\right) f\left(\tilde{y}_{2} \mid \mathcal{Y}_{1}\right) \cdots f\left(\tilde{y}_{T} \mid \mathcal{Y}_{T-1}\right)
$$

It follows from (9) that

$$
\tilde{y}_{t} \mid \mathcal{Y}_{t-1} \sim N\left(J_{t} Z a_{t \mid t-1}, \Omega_{t}\right)
$$

where

$$
\begin{aligned}
\Omega_{t} & =E\left[\left(\tilde{J}_{t} Z\left(\alpha_{t}-a_{t \mid t-1}\right)+\tilde{\varepsilon}_{t}\right)\left(\tilde{J}_{t} Z\left(\alpha_{t}-a_{t \mid t-1}\right)+\tilde{\varepsilon}_{t}\right)^{\prime}\right] \\
& =\tilde{J}_{t} Z V_{t \mid t-1} Z^{\prime} \tilde{J}_{t}^{\prime}+I_{N_{t}} \otimes \Sigma_{t},
\end{aligned}
$$

so the log likelihood of the observed sample is

$$
\ln L=-\frac{\sum_{t=1}^{T} N_{t}}{2} \ln (2 \pi)-\frac{1}{2} \sum_{t=1}^{T}\left[\ln \left|\Omega_{t}\right|+\left(\tilde{y}_{t}-\tilde{J}_{t} Z a_{t \mid t-1}\right)^{\prime} \Omega_{t}^{-1}\left(\tilde{y}_{t}-\tilde{J}_{t} Z a_{t \mid t-1}\right)\right] .
$$

As $\Omega_{t}$ is of dimension $m N_{t} \times m N_{t}$, calculation of $\left|\Omega_{t}\right|$ and $\Omega_{t}^{-1}$ directly is time consuming. However, the Appendix shows that we may rewrite (14) in a convenient form. First,

$$
\left|\Omega_{t}\right|=\left|\Sigma_{t}\right|^{N_{t}-G} \prod_{h=1}^{G}\left|\Lambda_{h}\right|
$$

\footnotetext{
${ }^{3}$ An alternative approach based on the EM algorithm is explored in an appendix to the working paper version of the paper (Lind 2004).
} 
where

$$
\Lambda_{h}:= \begin{cases}N_{1}^{g} J_{1} Z V_{t \mid t-1} Z^{\prime} J_{1}^{\prime}+\Sigma_{t} & \text { if } h=1 \\ N_{h}^{g} J_{h} Z\left[V_{t \mid t-1}^{-1}+\sum_{i=1}^{h-1} N_{i}^{g} Z^{\prime} J_{i}^{\prime} \Sigma_{t}^{-1} J_{i} Z\right]^{-1} Z^{\prime} J_{h}^{\prime}+\Sigma_{t} & \text { if } h>1\end{cases}
$$

Also,

$$
\begin{aligned}
\Psi_{t}: & =\left(\tilde{y}_{t}-\tilde{J}_{t} Z a_{t \mid t-1}\right)^{\prime} \Omega_{t}\left(\tilde{y}_{t}-\tilde{J}_{t} Z a_{t \mid t-1}\right) \\
= & \sum_{h=1}^{G} \operatorname{tr}\left[N_{h t}^{g} \Sigma^{-1} \operatorname{Cov}_{h t}\left(y_{i t}\right)\right] \\
& +\left(\bar{y}_{t}^{G}-Z a_{t \mid t-1}\right)^{\prime} \Xi_{t}\left\{I_{G m}-Z\left[V_{t \mid t-1}^{-1}+Z^{\prime} \Xi_{t} Z\right]^{-1} Z^{\prime} \Xi_{t}\right\}\left(\bar{y}_{t}^{G}-Z a_{t \mid t-1}\right) .
\end{aligned}
$$

where $N_{h t}^{g}$ is the number of members of group $h$ at data $t, \operatorname{Cov}_{h t}\left(y_{i t}\right)$ denotes the intragroup empirical variance-covariance matrix of the $y_{i t} \mathrm{~s}$ at date $t$ (without degrees of freedomadjustment), and $\Xi_{t}=\mathcal{N}^{G} \otimes \Sigma_{t}^{-1}$. From equations (15) and (16) we can then calculate the likelihood value

$$
\ln L=-\frac{\sum_{t=1}^{T} N_{t}}{2} \ln (2 \pi)-\frac{1}{2} \sum_{t=1}^{T}\left[\ln \left|\Omega_{t}\right|+\Psi_{t}\right]
$$

\section{Computational efficiency}

The main rationale for the new Kalman filtering recursion (10) and the likelihood function (17) is computational efficiency. Theoretically, one could use the traditional Kalman filter. However, as this algorithm requires inversion of $\tilde{J}_{t} Z V_{t \mid t-1} Z^{\prime} \tilde{J}_{t}^{\prime}+I_{N_{t}} \otimes \Sigma_{t}$, which is of dimension $m N_{t} \times m N_{t}$, which can be a large number, this is computationally costly.

Another alternative to the traditional Kalman filter would be the univariate approach where each element of the observation vector $\tilde{y}_{t}$ is processed sequentially. This approach was suggested by Anderson and Moore (1979, Section 6.4) and further developed by Durbin and Koopman (2001, Section 6.4) and Koopman and Durbin $(2000,2003)$. If every $\tilde{y}_{t}$ is $m N_{t} \times 1$, 
this leads to a Kalman recursion of length $m N_{t} T$, but where each step in the recursion only requires scalar divisions rather than matrix inversions. The recursions are hence longer than with the approach suggested in this paper, but each step is faster.

The recursion (10) requires inverting $V_{t \mid t-1}$ and $\left[V_{t \mid t-1}^{-1}+Z^{\prime}\left(N_{t}^{G} \otimes \Sigma_{t}^{-1}\right) Z\right]$, which are both of dimension $n \times n$, on each step of the recursion. However, as $n$ is a relatively small number in most cases, the computational burden of these inversions is not very large.

The computational burden of each of the three algorithms is easily seen to increase linearly in $T$, the number of periods, so increased $T$ has the same effect on all three. For increasing $N_{t}$, the standard Kalman algorithm will require inversion of a matrix of dimensionality proportional to $N_{t}$, so the computational cost is increasing at a faster than linear rate. The univariate approach requires a recursion whose length is increasing linearly in $N_{t}$ where the computational cost of each iteration is constant, so the computational burden is linear in $N_{t}$. Finally, the recursion (10) is non-increasing in $N_{t}$. This means that there is some sample size above which the recursion (10) will be more efficient than both the traditional Kalman filter and the univariate approach. However, this could be a sample size that is not relevant for practical purposes. The computational burden of the likelihood function follows a similar pattern.

Table 1 about here

To get a better grasp of the computational burden of the different algorithms, I have carried out some simple simulations. Table 1 reports the computing time for each of the three algorithms for different sample sizes on two models. In the first model, $\alpha_{t}$ follows a simple random walk so $\alpha_{t}=\alpha_{t-1}+\xi_{t}$ and $\alpha_{t}$ is a scalar. In the second model, $\alpha_{t}$ follows an $\operatorname{ARMA}(2,2)$ model and the dimension of $\alpha_{t}$ is $n=6$. All simulation are run using $T=100$ periods. Experimentation with other values of $T$ shows that the actual burden is very close 
to being linear in $T$ as predicted theoretically.

When $N_{t}=1$, the computational burden is quite similar for the three algorithms with the univariate approach being the most efficient. But even for $N_{t}$ relatively small, the algorithm presented herein becomes more efficient than the two others. However, the univariate approach is clearly more efficient than the traditional Kalman filter, whose computational burden gets very high for sample sizes of 100 and 1000. The results are very similar for calculation of the likelihood function.

\section{Conclusion}

We have presented a modified Kalman filtering algorithm to perform calculations on repeated surveys. The procedure makes it possible to obtain efficient estimates of underlying estimates of the laws of motion of the parameters of interest. By using the time series properties of repeated surveys, we get more precise estimates in each period than we would get by considering each survey separately. Hence even if each survey is of moderate size, we get reliable estimates, so we can produce estimates with higher frequency than what would be possible without using the time series approach.

Although the standard Kalman filter or preferably the univariate approach (Durbin and Koopman 2001, Koopman and Durbin 2003) may be used, this paper has presented an algorithm that is computationally superior as the traditional filtering implies inverting matrices of high dimensionality and the univariate approach leads to recursions with length increasing in the size of the sample of each survey.

A useful extension to the approach herein would be to extend the filtering and smoothing to handle diffuse initial conditions exactly as explored by e.g. Koopman and Durbin (2003). 
Finally, at the present stage, the method only admits estimation of sample means. An interesting extension would be to allow for estimation of repeated regression coefficients integrating the estimation of the regressions with the Kalman filter.

\section{References}

Anderson, B. D. O., and J. B. Moore (1979): Optimal Filtering. Englewood Cliffs: Prentice-Hall.

Binder, D. A. and J. P. Dick (1989): "Modelling and estimation for repeated surveys." Survey Methodology 15: 29-45.

Binder, D.A. and M. A. Hidiroglou (1988): "Sampling in time". In P.K. Krishnaiah and C.R. Rao (eds.): Handbook of Statistics, pp. 187-211.

Blight, B. J. N. and A. J. Scott (1973): "A stochastic model for repeated surveys." Journal of the Royal Statistical Society Series B, 35: 61-66.

de Jong, P. (1989): "Smoothing and interpolation with the state-space model." Journal of the American Statistical Association 84: 1085-88.

Durbin, J., and S. J. Koopman (2001): Time Series Analysis by State Space Methods. Oxford: Oxford University Press.

Gurney, M. and J. F. Daly (1965): "A multivariate approach to estimation in periodic sample surveys." American Statistical Association Proceedings of the Social Statistics Section 8: 242-57.

Hamilton, J. D. (1994): Time series analysis. Princeton: Princeton University Press. 
Harvey, A. C. (1989): Forecasting, structural time series and the Kalman filter. Cambridge: Cambridge University Press.

Harvey, A. C. and C.-H. Chung (1999): "Estimating the underlying change in UK unemployment." Journal of the Royal Statistical Society Series A 163: 303-39.

Jessen, R. J. (1942): "Statistical investigation of a farm survey for obtaining farm facts." Iowa Agricultural Experimental Station Research Bulletin 304: 54-59.

Kohn, R., and C. F. Ansley (1989): "A fast algorithm for signal extraction, influence and cross-validation in state space models." Biometrika 76: 65-79.

Koopman, S. J., and J. Durbin (2003): "Fast filtering and smoothing for multivariate state space models" Journal of Time Series Analysis 21: 281-96.

Koopman, S. J., and J. Durbin (2003): "Filtering and smoothing of state vector for diffuse state-space models." Journal of Time Series Analysis 24: 85-98.

Lind, J. T. (2004): "Repeated surveys and the Kalman filter." Memorandum, Department of Economics, University of Oslo.

Pfeffermann, D. (1991): "Estimation and seasonal adjustment of population means using data from repeated surveys." Journal of Business and Economic Statistics 9, 163-77.

Scott, A. J. and T. M. F. Smith (1974): "Analysis of repeated surveys using time series methods." Journal of the American Statistical Association 69: 647-78.

Tam, S. M. (1987): "Analysis of repeated surveys using a dynamic linear model." 


\section{Appendix: Proofs}

This Appendix outlines the idea behind the proofs of he main results of the paper. More detailed proofs are found in the working paper version (Lind 2004), and are also available upon request.

\section{Proof of equation (10)}

First, the matrix inversion lemma gives

$$
\begin{aligned}
& \left(\tilde{J}_{t} Z V_{t \mid t-1} Z^{\prime} \tilde{J}_{t}^{\prime}+I_{N_{t}} \otimes \Sigma_{t}\right)^{-1} \\
= & I_{N_{t}} \otimes \Sigma_{t}^{-1}-I_{N_{t}} \otimes \Sigma_{t}^{-1} \tilde{J}_{t} Z\left(V_{t \mid t-1}^{-1}+Z^{\prime} \tilde{J}_{t}^{\prime}\left(I_{N_{t}} \otimes \Sigma_{t}^{-1}\right) \tilde{J}_{t} Z\right)^{-1} Z^{\prime} \tilde{J}_{t}^{\prime}\left(I_{N_{t}} \otimes \Sigma_{t}^{-1}\right) .
\end{aligned}
$$

Also, $\tilde{J}_{t}^{\prime}\left(I_{N_{t}} \otimes \Sigma_{t}^{-1}\right) \tilde{J}_{t}=\sum_{i=1}^{N_{t}} \tilde{J}_{g(i) t}^{\prime} \Sigma^{-1} \tilde{J}_{g(i) t}=\mathcal{N}_{t}^{G} \otimes \Sigma^{-1}$, where $\mathcal{N}_{t}^{G}=\operatorname{diag}\left(N_{1 t}^{g}, \ldots, N_{G t}^{g}\right)$ for $N_{h t}^{g}$ the number of members in group $h$ at time $t$. Substituting from (18) and using the matrix inversion lemma a second time, the Kalman gain becomes

$$
K_{t}=\left(V_{t \mid t-1}^{-1}+Z^{\prime}\left(\mathcal{N}^{G} \otimes \Sigma^{-1}\right) Z\right)^{-1} Z^{\prime} \tilde{J}_{t}^{\prime}\left(I_{N_{t}} \otimes \Sigma^{-1}\right)
$$

SO

$$
a_{t \mid t}-a_{t \mid t-1}=\left(V_{t \mid t-1}^{-1}+Z^{\prime}\left(\mathcal{N}^{G} \otimes \Sigma^{-1}\right) Z\right)^{-1} Z^{\prime} \tilde{J}_{t}^{\prime}\left(I_{N_{t}} \otimes \Sigma^{-1}\right)\left(\tilde{y}_{t}-\tilde{J}_{t} Z a_{t \mid t-1}\right) .
$$

We also have

$$
\tilde{J}_{t}^{\prime}\left(I_{N_{t}} \otimes \Sigma^{-1}\right)\left(\tilde{y}_{t}-\tilde{J}_{t} Z a_{t \mid t-1}\right)=\left(\mathcal{N}_{t}^{G} \otimes \Sigma^{-1}\right)\left(\bar{y}_{t}^{G}-Z a_{t \mid t-1}\right)
$$


where $\bar{y}_{t}^{G}$ was defined in (11) and we used the fact that $\left(J_{1}^{\prime}, \ldots J_{G}^{\prime}\right)^{\prime}=I_{G m}$. Consequently, the Kalman updating becomes

$$
a_{t \mid t}=a_{t \mid t-1}+\left(V_{t \mid t-1}^{-1}+Z^{\prime}\left(\mathcal{N}_{t}^{G} \otimes \Sigma^{-1}\right) Z\right)^{-1} Z^{\prime}\left(\mathcal{N}^{G} \otimes \Sigma^{-1}\right)\left(\bar{y}_{t}^{G}-Z a_{t \mid t-1}\right) .
$$

The expression for updating the covariance simplifies to the variance formula (10a), and then (20) simplifies to (10b).

\section{Proof of equation (15)}

Assume without loss of generality that $\tilde{y}_{t}$ is constructed such that the first $m N_{1 t}^{g}$ elements belong to group 1, the following $m N_{2 t}^{g}$ elements to group 2 and so on. Define for each group $h \in(1, \ldots, G)$ the vector $J_{h}^{g}=\mathbf{1}_{N_{h}^{g} \times 1} \otimes J_{h}$. For simplicity of notation, we omit the subscript $t$ for matrices that depend on group sizes throughout this and the following Section. Now $E\left(\tilde{y}_{t} \mid \mathcal{Y}_{t-1}\right)=\left(\begin{array}{lll}J_{1}^{g \prime} & \cdots & J_{G}^{g \prime}\end{array}\right)^{\prime} Z a_{t \mid t-1}$. Then the upper left $m N_{1}^{g} \times m N_{1}^{g}$-block of $\Omega_{t}$ contains the covariance of the elements from group 1 ; call this sub-matrix $\Omega_{t}^{1: 1}$. The upper left $m\left(N_{1}^{g}+N_{2}^{g}\right) \times m\left(N_{1}^{g}+N_{2}^{g}\right)$-block contains the covariance between the elements from group 1 and 2 ; call this sub-matrix $\Omega_{t}^{1: 2}$. Generally, the covariance matrix of the elements belonging to group 1 to $h$ is $\Omega_{t}^{1: h}=J_{1: h}^{g} Z V_{t \mid t-1} Z^{\prime} J_{1: h}^{g \prime}+I_{\left(N_{1}^{g}+\ldots+N_{h}^{g}\right)} \otimes \Sigma_{t}$ where $J_{1: h}^{g}=\left(\begin{array}{ccc}J_{1}^{g \prime} & \cdots & J_{h}^{g \prime}\end{array}\right)^{\prime}$. Hence for each $h \geq 1$

$$
\Omega_{t}^{1: h+1}=\left(\begin{array}{cc}
\Omega_{t}^{1: h} & J_{1: h}^{g} Z V_{t \mid t-1} Z^{\prime} J_{h+1}^{g \prime} \\
J_{h+1}^{g} Z V_{t \mid t-1} Z J_{1: h}^{g \prime} & J_{h+1}^{g} Z V_{t \mid t-1} Z^{\prime} J_{h+}^{g \prime}+I_{N_{h+1}^{g}} \otimes \Sigma_{t}
\end{array}\right),
$$

which means that

$$
\left|\Omega_{t}^{1: h+1}\right|=\left|\Omega_{g}^{1: h}\right|\left|J_{h+1}^{g} Z V_{t \mid t-1} Z^{\prime} J_{h+}^{g \prime}+I_{N_{h+1}^{g}} \otimes \Sigma_{t}-J_{1: h}^{g} Z V_{t \mid t-1} Z^{\prime} J_{h+1}^{g \prime}\left(\Omega_{t}^{1: h}\right)^{-1} J_{h+1}^{g} Z V_{t \mid t-1} Z J_{1: h}^{g \prime}\right| .
$$


Furthermore, using the matrix inversion lemma on $\left(\Omega_{t}^{1: h}\right)^{-1}$, some manipulations yield

$$
\begin{aligned}
& J_{h+1}^{g} Z V_{t \mid t-1} Z^{\prime} J_{h+1}^{g \prime}+I_{N_{h+1}^{g}} \otimes \Sigma_{t}-J_{1: h}^{g} Z V_{t \mid t-1} Z^{\prime} J_{h+1}^{g \prime} \Omega_{p}^{-1} J_{h+1}^{g} Z V_{t \mid t-1} Z^{\prime} J_{1: h}^{g \prime} \\
= & J_{h+1}^{g} Z\left[V_{t \mid t-1}^{-1}+Z^{\prime} J_{1: h}^{g \prime}\left(I_{p} \otimes \Sigma_{t}^{-1}\right) J_{1: h}^{g} Z\right]^{-1} Z^{\prime} J_{h+1}^{g \prime}+I_{N_{h+1}^{g}} \otimes \Sigma_{t} .
\end{aligned}
$$

A Gauss-Jordan transformation of this expression gives us

$$
\begin{aligned}
& \left|J_{h+1}^{g} Z\left[V_{t \mid t-1}^{-1}+Z^{\prime} J_{1: h}^{g \prime}\left(I_{p} \otimes \Sigma_{t}^{-1}\right) J_{1: h}^{g} Z\right]^{-1} Z^{\prime} J_{h+1}^{g \prime}+I_{N_{h+1}^{g}} \otimes \Sigma_{t}\right| \\
= & \left|\Sigma_{t}\right|^{N_{h+1}^{g}-1}\left|N_{h+1}^{g} J_{h+1} Z\left[V_{t \mid t-1}^{-1}+Z^{\prime} J_{1: h}^{g \prime}\left(I_{p} \otimes \Sigma_{t}^{-1}\right) J_{1: h}^{g} Z\right]^{-1} Z^{\prime} J_{h+1}^{\prime}+\Sigma_{t}\right| .
\end{aligned}
$$

Substituting into (21), we get

$$
\left|\Omega_{t}^{1: h+1}\right|=\left|\Omega_{t}^{1: h}\right|\left|\Sigma_{t}\right|^{N_{h+1}^{g}-1}\left|N_{h+1}^{g} J_{h+1} Z\left[V_{t \mid t-1}^{-1}+\sum_{i=1}^{h} N_{i}^{g} Z^{\prime} J_{i}^{\prime} \Sigma_{t}^{-1} J_{i} Z\right]^{-1} Z^{\prime} J_{h+1}^{\prime}+\Sigma_{t}\right| .
$$

Furthermore,

$$
\begin{aligned}
\left|\Omega_{t}^{11}\right| & =\left|\mathbf{1}_{N_{1}^{g} \times N_{1}^{g}} \otimes J_{1} Z V_{t \mid t-1} Z^{\prime} J_{1}^{\prime}+I_{N_{1}^{g}} \otimes \Sigma_{t}\right| \\
& =\left|\Sigma_{t}\right|^{N_{1}^{g}-1}\left|N_{1}^{g} J_{1} Z V_{t \mid t-1} Z^{\prime} J_{1}^{\prime}+\Sigma_{t}\right| .
\end{aligned}
$$

Consequently, we may rewrite $\left|\Omega_{t}\right|$ as

$$
\left|\Omega_{t}\right|=\left|\Sigma_{t}\right|^{N_{t}-G}\left|N_{1}^{g} J_{1} Z V_{t \mid t-1} Z^{\prime} J_{1}^{\prime}+\Sigma_{t}\right| \prod_{h=2}^{G}\left|N_{h}^{g} J_{h} Z\left[V_{t \mid t-1}^{-1}+\sum_{i=1}^{h-1} N_{i}^{g} Z^{\prime} J_{i}^{\prime} \Sigma_{t}^{-1} J_{i} Z\right]^{-1} Z^{\prime} J_{h}^{\prime}+\Sigma_{t}\right| .
$$

\section{Proof of equation (16)}

First, as $y_{i t}-J_{g(i)} Z a_{t \mid t-1}=\left(y_{i t}-\bar{y}_{g(i) t}\right)+\left(\bar{y}_{g(i) t}-J_{g(i)} Z a_{t \mid t-1}\right)$ where $\bar{y}_{g t}$ is the average value of $y$ in group $g$ at date $t$, we get

$$
\begin{aligned}
& \left(\tilde{y}_{t}-\tilde{J}_{t} Z a_{t \mid t-1}\right)^{\prime}\left(I_{N_{t}} \otimes \Sigma_{t}^{-1}\right)\left(\tilde{y}_{t}-\tilde{J}_{t} Z a_{t \mid t-1}\right) \\
= & \sum_{g=1}^{G} \operatorname{tr}\left[N_{g}^{g} \Sigma^{-1} \operatorname{Cov}_{g t} y_{i t}\right]+\left(\bar{y}_{t}^{G}-Z a_{t \mid t-1}\right)^{\prime}\left(\mathcal{N}^{G} \otimes \Sigma_{t}^{-1}\right)\left(\bar{y}_{t}^{G}-Z a_{t \mid t-1}\right)
\end{aligned}
$$


where the last line uses the fact that the trace of a scalar is the scalar. Furthermore

$$
\begin{aligned}
& \left(\tilde{y}_{t}-\tilde{J}_{t} Z a_{t \mid t-1}\right)^{\prime}\left(I_{N_{t}} \otimes \Sigma_{t}^{-1}\right) \tilde{J}_{t} Z\left[V_{t \mid t-1}^{-1}+Z^{\prime} \tilde{J}_{t}^{\prime}\left(I_{N_{t}} \otimes \Sigma_{t}^{-1}\right) \tilde{J}_{t} Z\right]^{-1} \\
& \times Z^{\prime} \tilde{J}_{t}^{\prime}\left(I_{N_{t}} \otimes \Sigma_{t}^{-1}\right)\left(\tilde{y}_{t}-\tilde{J}_{t} Z a_{t \mid t-1}\right) \\
= & \left(\bar{y}_{t}^{G}-Z a_{t \mid t-1}\right)^{\prime}\left(\mathcal{N}^{G} \otimes \Sigma_{t}^{-1}\right) Z\left[V_{t \mid t-1}^{-1}+Z^{\prime} \tilde{J}_{t}^{\prime}\left(I_{N_{t}} \otimes \Sigma_{t}^{-1}\right) \tilde{J}_{t} Z\right]^{-1} \\
& \times Z^{\prime}\left(\mathcal{N}^{G} \otimes \Sigma_{t}^{-1}\right)\left(\bar{y}_{t}^{G}-Z a_{t \mid t-1}\right) .
\end{aligned}
$$

Consequently by using the matrix inversion lemma on $\Omega_{t}$ we get

$$
\begin{aligned}
& \Psi_{t}=\sum_{g=1}^{G} \operatorname{tr}\left[N_{g}^{g} \Sigma^{-1} \operatorname{Cov}_{g t} y_{i t}\right] \\
& +\left(\bar{y}_{t}^{G}-Z a_{t \mid t-1}\right)^{\prime}\left(\mathcal{N}^{G} \otimes \Sigma_{t}^{-1}\right)\left\{I_{G m}-Z\left[V_{t \mid t-1}^{-1}+Z^{\prime}\left(\mathcal{N}^{G} \otimes \Sigma_{t}^{-1}\right) Z\right]^{-1}\right. \\
& \left.\times Z^{\prime}\left(\mathcal{N}^{G} \otimes \Sigma_{t}^{-1}\right)\right\}\left(\bar{y}_{t}^{G}-Z a_{t \mid t-1}\right) .
\end{aligned}
$$


Table 1: Comparisons of the computational burden of different algorithms

\begin{tabular}{|c|c|c|c|c|c|c|}
\hline \multirow[b]{2}{*}{$\mathrm{N}_{\mathrm{t}}$} & \multicolumn{3}{|c|}{ Filtering } & \multicolumn{3}{|c|}{ Likelihood } \\
\hline & $\begin{array}{c}\text { Kalman } \\
\text { filter }\end{array}$ & $\begin{array}{c}\text { Univariate } \\
\text { approach }\end{array}$ & $\begin{array}{c}\text { Repeated } \\
\text { surveys }\end{array}$ & $\begin{array}{l}\text { Kalman } \\
\text { filter }\end{array}$ & $\begin{array}{c}\text { Univariate } \\
\text { approach }\end{array}$ & $\begin{array}{c}\text { Repeated } \\
\text { surveys }\end{array}$ \\
\hline \multicolumn{7}{|c|}{ Random walk } \\
\hline 1 & 0.61 & 0.31 & 0.41 & 0.56 & 0.23 & 0.69 \\
\hline 5 & 0.94 & 1.24 & 0.42 & 1.22 & 1.14 & 0.67 \\
\hline 10 & 1.56 & 2.42 & 0.41 & 2.03 & 2.18 & 0.70 \\
\hline 100 & 136 & 29.1 & 0.47 & 219 & 26.6 & 0.78 \\
\hline 1000 & 244860 & 233 & 0.47 & 381720 & 281 & 0.78 \\
\hline \multicolumn{7}{|c|}{$\operatorname{ARMA}(2,2)$} \\
\hline 1 & 1.44 & 0.83 & 0.97 & 0.63 & 0.34 & 1.39 \\
\hline 5 & 1.92 & 4.48 & 1.41 & 1.52 & 2.53 & 1.80 \\
\hline 10 & 3.28 & 10.44 & 1.52 & 3.28 & 5.16 & 1.84 \\
\hline 100 & 156 & 102 & 1.60 & 219 & 63 & 3.10 \\
\hline 1000 & 259660 & 1091 & 1.50 & 358280 & 546 & 3.20 \\
\hline
\end{tabular}

Table entries give the computing time in milliseconds for the three estimators for different sample sizes of each survey. "Kalman filter" is the traditional Kalman filter, "Univariate approach" is the Koopman and Durbin (2000, 2003) algorithm, and "Repeated surveys" is the algorithms presented in this paper. Computation time of the likelihood function excludes the required prior run of the filter. 